\title{
The Effects of Sorption-Desorption Cycling on the Anisotropy of Mechanical Properties at the Local Crystalline Orientation of a Polycrystalline 316L Stainless Steel Plasma Nitrided
}

\author{
GARZÓN Carlos Mario ${ }^{1 *}$, FISHER Rafael R. ${ }^{2}$ and RECCO Abel A. ${ }^{2}$ \\ 1. Departamento de Física, Universidad Nacional de Colombia, Bogotá, Colombia. \\ 2. Laboratório de Física de Plasma - LabPlasma, Departamento de Física, Universidade do Estado de \\ Santa Catarina, Joinville, Brasil. \\ * Corresponding author: cmgarzono@unal.edu.co
}

Mesoscale plasticity anisotropy of nitrogen bearing steel structures in wear tests has been reported [1]. The actual relationship between local deformation mode, wear constraints and individual grain orientation is being studied, whit not conclusive experimental-theoretical formulation [1-3].

The relationship between individual grain orientation and local mechanical properties (hardness and Young's modulus) in samples of polycrystalline 316L stainless steel plasma nitrided is studied through electron backscatter diffraction (EBSD) in a field emission scanning electron microscope (FEG-SEM) and an atomic force microscope (AFM). This contribution aims at assessing the effect of cycling periods of nitrogen absorption (gas atmosphere $75 \%$-vol. $\mathrm{H}_{2}+25 \%$-vol. $\mathrm{N}_{2}$ ) and of nitrogen desorption (gas atmosphere $75 \%$-vol. $\mathrm{H}_{2}+25 \%$-vol. Ar) on the anisotropy of mechanical properties at the scale of individual grains.

Samples were nitrided by a total time of 90 minutes, alternating sorption-desorption cycles of equal duration among them. We observed that as-nitrided virgin surfaces were not suitable for EBSD characterization, due to intense surface roughening, which was induced by the nitriding process itself. Thus, an experimental polishing procedure, that is comprised of early grinding in $\mathrm{Al}_{2} \mathrm{O}_{3}$ slurries and late polishing in colloidal silica was developed, which allows preparing the nitrided region for crystallographic analysis via EBSD. The suitability of the polished surfaces for conducting EBSD characterization was assessed through an analysis of both the surface roughness (appraised by atomic force microscopy) and the quality of the Kikuchi diffraction patterns.

Through AFM profiling inside fiducial Berkovich imprints a resolution of $100 \mathrm{~nm}$ in the total removed layer was attainable via careful control of the polishing parameters. Polished surfaces were obtained after removing layers as thin as $1.4 \mu \mathrm{m}$ ( $15 \%$ of the total nitrided case). The maximal angular deviation from parallelism between the polished and the original surfaces was around $0.1^{\circ} \pm 0.05^{\circ}$, which is one magnitude order better than the traditional sample preparation.

Hough space associated with the Kikuchi diffraction patterns showed a somewhat lack of resolution in the nitrided case, with regard to the non-nitrided material. This was addressed to nitrided-induced work-hardening effects.

It was possible to appraise the hardness and Young modulus of the crystals with the following textures $\left( \pm 5^{\circ}\right)$, regarding the sample surface parallel to: (100), (101) or (111). In both, nitrogen-lean and nitrided samples, the Young modules was lower in grains with texture (100)||surface and higher in grains with texture (111)||surface. On the other hand, hardness was dependent on the actual sorption-desorption cycle. In general, grains with texture (111)||surface were harder, although in samples nitrided with one sorption + one desorption stage those grains (111) were softer. With EBSD results as a basis, we suggest that the plastic deformation state does not affect the Youngs modulus while hardness is indeed affected. Nitriding induced rotations of grain texture due to plastic deformation, which influenced the actual hardness of individual grain orientations. 
References:

[1] D H Mesa, C M Garzón and A P Tschiptschin, Wear, 267 (2009), 99-103.

[2] J Stella et al., Wear, 316 (2014), 1-5.

[3] D H Mesa, C M Garzón and A P Tschiptschin, Wear, 271 (2011), 1372-1377.

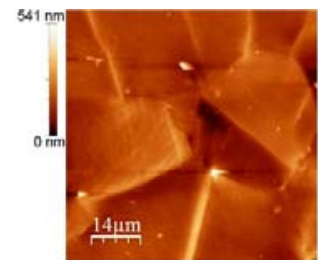

Unpolished, as-nitrided 1.

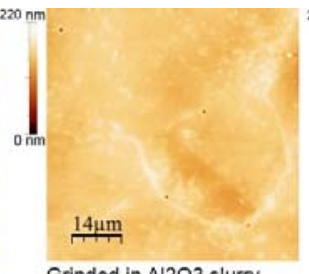

Grinded in $\mathrm{Al} 2 \mathrm{O} 3$ slurry

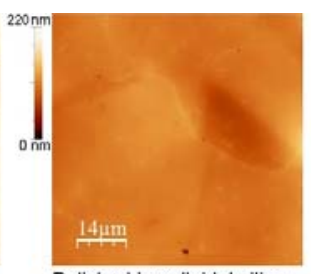

Polished in colloidal silica

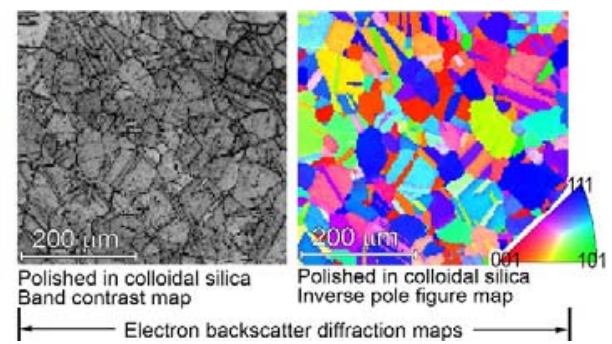

Figure 1. Atomic force micrographs (left) and EBSD maps (right) of the same microregion of one nitrided sample through the diverse stages of sample preparation and analysis.

FEG-SEM micrograph

Inverse pole figure superimposed on FEG-SEM micrograph

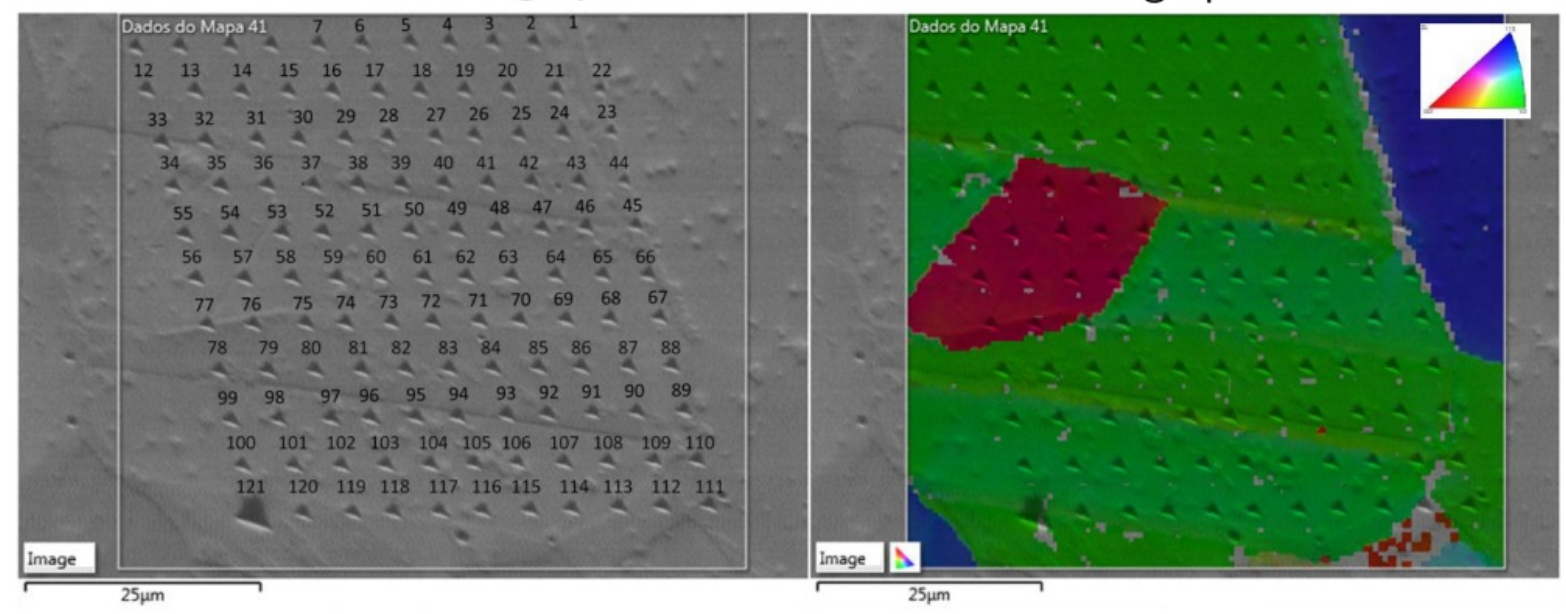

\begin{tabular}{|ccc|ccc|ccc|}
\hline Description & $\begin{array}{c}\text { Sorption } \\
\text { stages }\end{array}$ & $\begin{array}{c}\text { Desorption } \\
\text { stages }\end{array}$ & \multicolumn{3}{c|}{ Hardness } & \multicolumn{3}{c|}{ Young modulus } \\
Annealed, & \multicolumn{2}{c|}{ Non-nitrided } & $3.1 \pm 0.2$ & $3.4 \pm 0.1$ & $3.6 \pm 0.2$ & $201 \pm 26$ & $211 \pm 26$ & $217 \pm 27$ \\
nitrogen-lean & 1 & 0 & $14.4 \pm 0.4$ & $14.4 \pm 0.3$ & $14.8 \pm 0.4$ & $217 \pm 6$ & $237 \pm 7$ & $248 \pm 10$ \\
& 1 & 1 & $13.1 \pm 0.4$ & $13.0 \pm 0.3$ & $12.4 \pm 0.4$ & $217 \pm 6$ & $244 \pm 8$ & $246 \pm 10$ \\
Plasma nitrided & 3 & 3 & $13.8 \pm 0.3$ & $13.9 \pm 0.3$ & $14.8 \pm 0.3$ & $214 \pm 7$ & $239 \pm 8$ & $245 \pm 7$ \\
& 3 & & & & & & &
\end{tabular}

Figure 2. FEG-SEM micrograph (top-left) and IPF (right) of one microregion indented in the AFM microscope. Mechanical properties (bottom) as a function of grain microtexture. 